\title{
Damage Mitigating Controller Design for Structural Durability
}

\author{
Ravindra Patankar and Asok Ray
}

\begin{abstract}
Synthesis of a damage-mitigating control law requires additional information on damage states beyond what is needed for the design of a conventional output feedback controller. In this context, this brief paper establishes the necessity of a fatigue damage model that must account for the impact of variable-amplitude stress excitation on crack growth rate (e.g., crack retardation and sequence effects). It is shown that predicted structural durability and the damage-mitigating controller design could be grossly inaccurate if the fatigue crack damage model does not represent the effects of variable-amplitude cyclic stress. A specific example is given based on the design of output-feedback damage-mitigating controllers for a reusable rocket engine that was reported in an earlier publication. Simulation results are presented in this paper to compare the predicted structural durability and closed-loop performance of the rocket engine under the same controllers for two different damage models, with and without consideration of the effects of variable-amplitude stress.
\end{abstract}

Index Terms - Damage-mitigating control, fatigue crack growth, variable-amplitude stress excitation.

\section{INTRODUCTION}

The goal of damage-mitigating control (DMC) is to achieve a tradeoff between structural durability of selected critical components and overall dynamic performance of complex mechanical systems (e.g., aircraft, spacecraft, and energy conversion systems). Typically, a controller synthesis procedure guarantees stability of a closed-loop system within specified uncertainty bounds and, at the same time, ensures that the performance specifications are satisfied. Performance is usually defined in terms of command signal tracking, disturbance rejection, and/or control effort minimization. However, in general, the dynamics of material degradation occurring in critical plant components that are vulnerable to excessive structural stresses is not explicitly taken into account in the synthesis of control laws. The notion of DMC is to realize these two interrelated concepts in an integrated manner by synergistic combination of the disciplines of systems sciences and mechanics of materials. The earlier work reported by [6] and [11] shows that, using optimized open-loop feedforward control sequences, it is possible to substantially reduce the damage rate and accumulation in critical plant components with no significant loss in the dynamic performance.

The present state-of-the-art of DMC synthesis involves: 1) frequency-dependent weighting of selected plant variables

Manuscript received August 15, 1997; revised August 22, 1998. Recommended by Associate Editor, H. Jenkins. This work was supported in part by the National Science Foundation under Research Grants DMI-9424587 and CMS-9531835; and NASA Lewis Research Center under Grants NAG3-1673 and NAG-3-2016.

The authors are with the Department of Mechanical Engineering, The Pennsylvania State University, University Park, PA 16802 USA.

Publisher Item Identifier S 1063-6536(99)06454-4. which induce structural damage in critical plant components and 2) incorporation of these frequency-weighted variables as performance specifications along with traditional ones such as error-tracking of pertinent process variables. To realize performance/damage tradeoff for a reusable rocket engine, [4] synthesized a performance controller (i.e., designed with no consideration to damage) and a family of damage-mitigating controllers by taking fatigue damage of turbine blades into consideration. The robust linear controllers were designed via an induced L2-norm method applicable to sampled data systems. Reference [15] demonstrated the efficacy of a feedforward-feedback damage-mitigating controller on a laboratory test apparatus to simultaneously achieve life extension and enhanced performance. Reference [5] proposed a two-tier DMC system in which a linear robust controller at the lower tier followed a reference trajectory while a fuzzy controller at the upper layer made decisions for tradeoff between system performance and damage in critical components.

The fatigue crack damage model used in the above publications did not include the impact of variable-amplitude stresses on crack growth rate, i.e., crack retardation and sequence effects [14], [1]. Consequently, predicted damage and the damage-mitigating controller design could be grossly inaccurate. As an example, we consider the damage-mitigating controller for a reusable rocket engine reported by [4]. In this study, the beneficial effects of stress overload on structural integrity of turbine blades are ignored based on the erroneous assumption that the damage accumulation is monotonically increasing with peak stress, and thereby the damage caused by the performance controller was exaggerated. Unfortunately, this notion has been followed in the design of other damagemitigating controllers even though it may not always be correct under variable-amplitude stress excitation.

This brief paper establishes, for DMC design, the necessity of a damage model that must account for the impact of variable-amplitude stress excitation on crack growth rate (e.g., crack retardation and sequence effects). Recently, [9] have developed a state-space model of fatigue crack growth dynamics under cyclic stress excitation of variable amplitude. The state-space model is capable of capturing the effects of block loading, random loading, and irregular sequences including different combinations of single overload and underload. In the present paper, we use the state-space model of fatigue crack growth to reexamine the damage-mitigating controllers of a rocket engine [4]. The difference in structural durability of the engine turbine blades by the two different damage models is explained in view of the fatigue crack growth process associated with the closed-loop control system.

This paper is organized in six sections including the introduction. Section II provides a brief description of the reusable 
rocket engine under consideration. Section III presents an overview of the state-variable-based model of fatigue crack growth that has been used for quantitative evaluation of structural damage in the turbine blades. Section IV presents the procedure for synthesis of damage-mitigating controllers for the rocket engine. Results of simulation experiments are presented and discussed in Section V. Finally, the paper is summarized and concluded in Section VI.

\section{Description of the Reusable Rocket Engine}

This section presents a brief functional description of the bipropellant reusable rocket engine under consideration; details of operation and control of the rocket engine are reported by [12]. The propellants, namely, cryogenic hydrogen $\left(\mathrm{H}_{2}\right)$ fuel and cryogenic oxygen $\left(\mathrm{O}_{2}\right)$, are individually pressurized by separate closed-cycle turbopumps. Pressurized cryogenic fuel and oxygen are pumped into two high-pressure preburners that feed the respective hydrogen $\left(\mathrm{H}_{2}\right)$ and oxygen $\left(\mathrm{O}_{2}\right)$ turbines with fuel-rich hot gas. The fuel-rich exhaust gas from each turbine is injected into the main combustion chamber. The oxygen/hydrogen $\left(\mathrm{O}_{2} / \mathrm{H}_{2}\right)$ mixture ratio of 6.02 is maintained to make the most efficient use of the energy. The oxygen flow into each of the two preburners are independently controlled by the respective servo-valves while the valve position for oxygen flow into the main thrust chamber is held in a fixed position to derive the maximum possible power from the engine. The plant outputs of interest are oxygen/hydrogen $\left(\mathrm{O}_{2} / \mathrm{H}_{2}\right)$ mixture ratio and main combustion chamber pressure that are closely related to the rocket engine performance in terms of specific impulse, thrust-to-weight ratio, and combustion temperature. The plant model consists of twenty state variables, two control inputs, and two measured variables.

\section{Modeling of Fatigue Damage Dynamics}

This section briefly introduces the state-space model of fatigue crack under cyclic stress excitation of variable amplitude [9]. The model is formulated based on the crack closure concept [3] and is verified with experimental data [10], [13]. Unlike the existing crack growth models, the state-space model does not require a long history of stress excitation to calculate the crack opening stress and, therefore, savings in both computation time and memory requirement are significant. The effects of the cyclic stress history are captured by a fading memory model where the state equation for crack opening stress is a (piecewise) bilinear difference equation excited by the peaks and valleys of the current stress cycle and the previous stress cycle. This representation adequately captures the phenomena of crack retardation and acceleration resulting from block loading, random loading, and irregular sequences including different combinations of single overload and underload. The fatigue crack growth process is modeled as

$$
c_{k}=c_{k-1}+h\left(\Delta K_{k}^{\mathrm{eff}}\right), \quad \text { for } h(0)=0 ; k \geq 0 \text { and } c_{0}>0
$$

$\left.\Delta K_{k}^{\mathrm{eff}}=\right) \sqrt{\pi c_{k-1}} F c_{k-1}\left(S_{k}^{\max }-S_{k-\tau}^{o}\right)$

where the crack length $\left(c_{k}\right)$ at the end of the $k$ th cycle is a state variable; $h(\cdot)$ is a function defined by a lookup table in fracture mechanics [8]; and $F$ is the correction factor for finite geometry of the specimen. A cycle ranges from a minimum stress to the next immediate minimum stress. If the frequency and shape effects are negligible, the $k$ th stress cycle is defined by the maximum stress $S_{k}^{\max }$ and the following minimum stress $S_{k}^{\min }$. The delayed crack opening stress $S_{k-\tau}^{o}$ is used to calculate the crack growth during the $k$ th cycle where $\tau$ is the delay in number of cycles before the applied load actually becomes effective. When a cyclic loading is applied to a cracked specimen, cracked surfaces are farthest apart at maximum load but they come in contact as the load is reduced toward the minimum value. Upon reloading, the applied stress level at which the crack surfaces become fully open with no surface contact is called the crack opening stress $S^{\circ}$ [3]. As the crack opening stress $S^{\circ}$ is difficult to measure, such measurements have been made on only a few materials under a limited number of specimen geometry, material properties, and loading conditions. On the other hand, procedures available for analytically calculating $S^{o}$ under variable amplitude cyclic load are complicated, computationally intensive, and possibly inaccurate due to truncation of the loading history. Therefore, we have cast $S^{\circ}$ as a state variable so that no truncation of loading history is necessary for computations.

The two state variables used in the crack growth model are crack length $c$ and crack opening stress $S^{\circ}$. A first-order equation has been proposed to closely describe the dynamics of $S^{\circ}$ [9]. Some materials may show considerably delayed crack retardation after the application of an overload. For such materials, crack opening stress $S^{\circ}$ is delayed by a number of cycles, $\tau$, as seen in (2).

For computation of $S^{\circ}$ under constant amplitude stress in metallic materials, [7] has proposed an empirical relationship as a function of maximum stress $S^{\max }$, stress ratio $R \equiv$ $\left(S^{\min } / S^{\max }\right)$, constraint factor $\alpha$, specimen geometry, and flow stress $S^{\text {flow }}$. Reference [9] have used the steady-state value $S_{k}^{o s s}$ to construct a forcing function [7] that is capable of producing and experimentally validating a dynamic response for $S_{k}^{o}$ under variable-amplitude load

$$
\frac{S_{k}^{\text {oss }}}{S_{k}^{\max }}=A_{k}^{0}+A_{k}^{1} R_{k}+A_{k}^{2}\left(R_{k}\right)^{2}+A_{k}^{3}\left(R_{k}\right)^{3}
$$

where

$$
\begin{aligned}
& R_{k}=\frac{S_{k}^{\bmod }}{S_{k}^{\max }} ; \quad S_{k}^{\bmod }=\frac{\alpha S_{k}^{\min }+\alpha S_{k-1}^{\min }}{\alpha+1} \\
& A_{k}^{0}=\left(0.825-0.34 \alpha+0.05 \alpha^{2}\right)\left[\cos \left(\frac{\pi}{2} \frac{S_{k}^{\max } F}{S^{\text {flow }}}\right)\right]^{1 / \alpha} \\
& A_{k}^{1}=(0.415-0.071 \alpha)\left(\frac{S_{k}^{\text {max }} F}{S^{\text {flow }}}\right) \\
& A_{k}^{2}= \begin{cases}1-A_{k}^{0}-A_{k}^{1}-A_{k}^{3}, & \text { if } R_{k}>0 \\
0, & \text { if } R_{k} \leq 0\end{cases} \\
& A_{k}^{3}= \begin{cases}2 A_{k}^{0}+A_{k}^{1}-1, & \text { if } R_{k}>0 \\
0, & \text { if } R_{k} \leq 0\end{cases}
\end{aligned}
$$

and $\alpha$ is the constraint factor (one for plane strain and three for plane strain). Using $S_{k}^{o s s}$ in (3) as the forcing function, we propose the following constitutive relation in the form of a (piecewise) bilinear first-order difference equation for 
recursive computation of the crack opening stress $\left(S_{k}^{o}\right)$ at the completion of the $(k-1)$ th cycle

$$
\begin{aligned}
S_{k}^{o}= & \left(\frac{1}{1+\eta}\right) S_{k-1}^{o}+\left(\frac{\eta}{1+\eta}\right) S_{k}^{o s s}+\left(\frac{\lambda_{k}}{1+\eta}\right) \\
& \cdot\left(S_{k}^{o s s}-S_{k-1}^{o}\right) \Im\left(S_{k}^{o s s}-S_{k-1}^{o}\right)
\end{aligned}
$$

where the Heaviside function $\Im(x):=\left\{\begin{array}{l}0 \text { if } x \leq 0 \\ 1 \text { if } x>0\end{array}\right.$, and $S_{k}^{o s s}$ is the steady-state crack opening stress calculated from (3) if a constant amplitude stress $\left(S_{k}^{\max }, S_{k}^{\min }\right)$ was applied; the dimensionless decay parameter $\eta$ is dependent on the material as well as on the specimen thickness; and $\lambda_{k}:=\left(S_{k}^{\max }-\right.$ $\left.S_{k}^{\bmod }\right) /\left(S_{k}^{\max }-S_{k-1}^{\min }\right)$ is the dimensionless cycle-dependent pulse scaling factor. For 4340 steel (somewhat similar to gasturbine blade materials), decay parameter $\eta=0.001$ and delay parameter $\tau=250$.

Remark 1: The dynamic crack opening stress in (4) along with the difference equation (1) for crack increment $\left(c_{k+1}-c_{k}\right)$ during the $(k+1)$ th cycle constitutes the state-space model for fatigue crack growth.

Remark 2: The variable $S^{\text {oss }}$, generated from (3), is used to construct the (piecewise bilinear) forcing function in the dynamic (4). Under constant amplitude stress excitation, $S^{\text {oss }}$ is the steady-state solution of $S^{o}$. However, under variableamplitude stress excitation, $S^{\text {oss }}$ is different from the instantaneous crack opening stress $S_{k}^{o}$ and does not have any specific physical significance.

Remark 3: Following an overload cycle, the duration of crack retardation is represented by the dynamics of $S^{\circ}$ in the state-space model and hence controlled by the stressindependent decay parameter $\eta$ in (4). Physically, this duration depends on the ductility of the material which is controlled by heat treatment [13]. For example, a lower yield strength may produce a longer duration. Therefore, $\eta$ is possibly a function of material ductility.

Remark 4: It is desirable that $\eta$ be identified from experimental data under single-cycle overload stress excitation. However, in the absence of available experimental data, eta could be evaluated from the data generated by a predictive model such as FASTRAN-II [8] under variable-amplitude stress excitation.

\section{Synthesis of DAMAge-Mitigating OutPut FEEDBACK CONTROLLERS}

The main objective of damage-mitigating controller (DMC) synthesis is to achieve a tradeoff between system dynamic performance and structural durability. In the context of the rocket engine control, the dynamic performance is implemented as small tracking errors of the thrust chamber pressure and the oxygen/hydrogen $\left(\mathrm{O}_{2} / \mathrm{H}_{2}\right)$ mixture ratio, and structural durability as low fatigue damage rate and accumulation in the $\mathrm{O}_{2}$ and $\mathrm{H}_{2}$ turbine blades. These objectives are accomplished by appropriate selection of the (frequency-dependent) weighting functions for the controller synthesis. This section presents the design of a performance controller and a family of damage controllers. The performance controller is designed without taking damage into consideration. In essence, it is a conventional performance controller (PC).

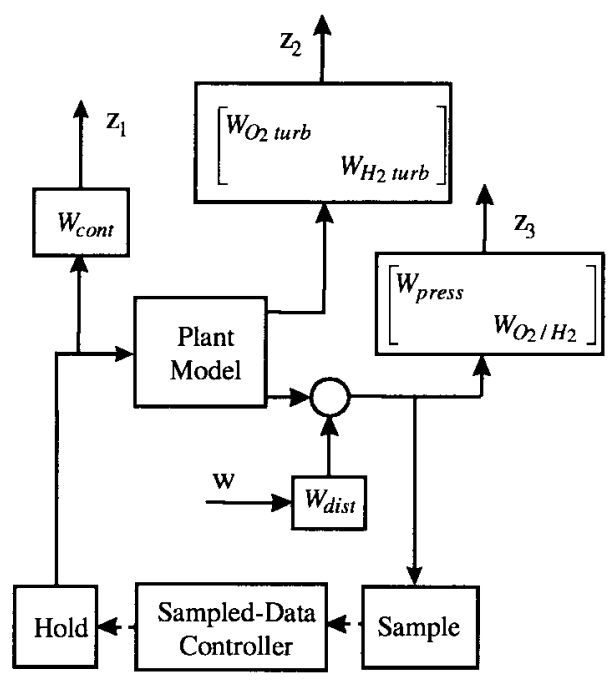

Fig. 1. Synthesis of the sampled-data controller.

The linear nominal plant model used for the controllerdesign is obtained by linearization of the nonlinear model of the reusable rocket engine at a chamber pressure of $17.58 \mathrm{MPa}$ $\left(2550 \mathrm{lbf} / \mathrm{in}^{2}\right)$ which represents the midpoint of the pressure ramp-up range from $14.48 \mathrm{MPa}\left(2100 \mathrm{lbf} / \mathrm{in}^{2}\right)$ to $20.69 \mathrm{Mpa}$ (3000 lbf/in ${ }^{2}$ ). Referring to Fig. 1, the design goal is to find a stabilizing discrete-time controller such that the induced $L_{2}$-norm of the transfer matrix from $W$ to $\left[\begin{array}{lll}z_{1} & z_{2} & z_{3}\end{array}\right]^{T}$ is minimized. We use the method of [2] for $L_{2}$-induced synthesis for sampled-data systems which makes use of a lifting technique which takes intersample behavior into account in the controller synthesis procedure. The sampled-data problem is recast in terms of an equivalent discrete-time $H_{\infty}$-synthesis problem.

Identical to the rocket engine controller design reported by [4], frequency-dependent performance weighting functions that penalize deviations of the actual thrust chamber pressure and $\mathrm{O}_{2} / \mathrm{H}_{2}$ mixture ratio from their reference trajectories are selected as

$$
W_{\text {press }}(s)=\frac{k_{p}\left(s+\alpha_{p}\right)}{s+\beta_{p}} \quad \text { and } \quad W_{\mathrm{O}_{2} / \mathrm{H}_{2}}(s)=k_{o}
$$

where

$$
\begin{aligned}
\alpha_{p}= & 7.5 ; \quad \beta_{p}=30.0 ; \quad k_{p}=2000.0 \\
k_{o}= & 1.0 \times 10^{5} ; \quad \text { for the damage-mitigating } \\
& \text { controller\#1 (DMC } 1) \\
\alpha_{p}= & 7.5 ; \quad \beta_{p}=30.0 ; \quad k_{p}=4000.0 \\
k_{o}= & 1.0 \times 10^{5} ; \quad \text { for the damage-mitigating } \\
& \text { controller\#2(DMC } 2) \\
\alpha_{p}= & 7.5 ; \quad \beta_{p}=30.0 ; \quad k_{p}=8000.0 \\
k_{o}= & 2.0 \times 10^{5} ; \quad \text { for the damage-mitigating } \\
& \text { controller\#3(DMC } 3) \\
\alpha_{p}= & 0.5 ; \quad \beta_{p}=1.0 ; \quad k_{p}=5000.0 \\
k_{o}= & 2.0 \times 10^{5} ; \quad \text { for the performance controller } \\
& (\mathrm{PC}) \text { and the Laplace transform variable } \\
& s \text { is in units of second } d^{-1} .
\end{aligned}
$$




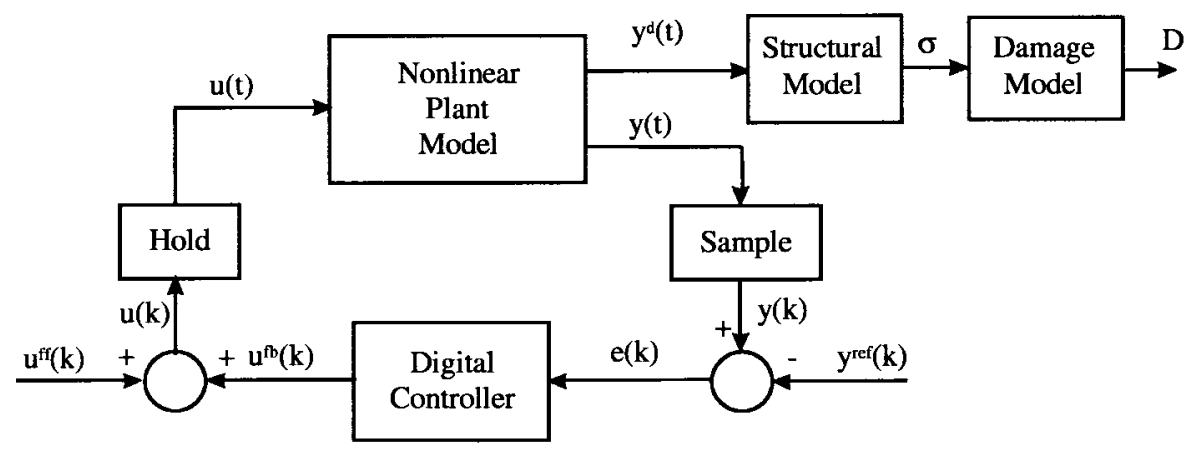

Fig. 2. Simulation block diagram.

A uniform scaling across the frequency spectrum is chosen for the $\mathrm{O}_{2} / \mathrm{H}_{2}$ ratio performance weight because deviations from the nominal value of 6.02 are undesirable at all frequencies, i.e., during both transient and steady-state operations. Also, each of the two reference signals is modeled as a disturbance input with the frequency-dependent weight

$$
W_{\text {dis }}(s)=\frac{0.001}{s+0.001} .
$$

Shaft torques generated by the oxidizer $\left(\mathrm{O}_{2}\right)$ and fuel $\left(\mathrm{H}_{2}\right)$ turbines are weighted in the syntheses of damage-mitigating controllers in an effort to reduce high-frequency transients that may cause excessive fatigue damage in the turbine blades. In theory, specifications of these weights along with the performance weights determine the tradeoff between dynamic performance and structural durability. For the damage controller design in this paper, these weights are selected as

$$
\begin{aligned}
W_{\mathrm{H}_{2} \text { turb }}(s) & =5 \frac{\left(s+2 \times 10^{-4}\right)}{s+0.1} \text { and } \\
W_{\mathrm{O}_{2} \text { turb }}(s) & =50 \frac{\left(s+2 \times 10^{-5}\right)}{s+0.1} .
\end{aligned}
$$

To achieve the ideal $\mathrm{O}_{2} / \mathrm{H}_{2}$ ratio performance, the $\mathrm{O}_{2}$ turbine must have a larger bandwidth and, therefore, the high frequency vibrations would be stronger than those on the $\mathrm{H}_{2}$ turbine. To reduce these vibrations, the weight on the $\mathrm{O}_{2}$ turbine is made larger than that for the $\mathrm{H}_{2}$ turbine at higher frequencies.

For the PC, the weights on the turbine torques in (7) are not used; however, weights on the control inputs are included to circumvent actuator saturation

$$
W_{\text {cont }}(s)=50000 \frac{s+0.02}{s+0.1} .
$$

For the DMC, the weights on torques serve to mitigate control inputs and therefore helps prevent actuator saturation. High-frequency control inputs are needed to generate instantaneous torques from the turbines for driving the pumps which must meet the large bandwidth transients of $\mathrm{O}_{2}$ and $\mathrm{H}_{2}$ mass flow-rates. If the torque at high frequencies is heavily attenuated, control efforts at these frequencies are reduced and consequently control input weighting are found to be unnecessary. In general, different sets of the turbine torque weights and performance weights lead to different performance and structural durability tradeoffs.

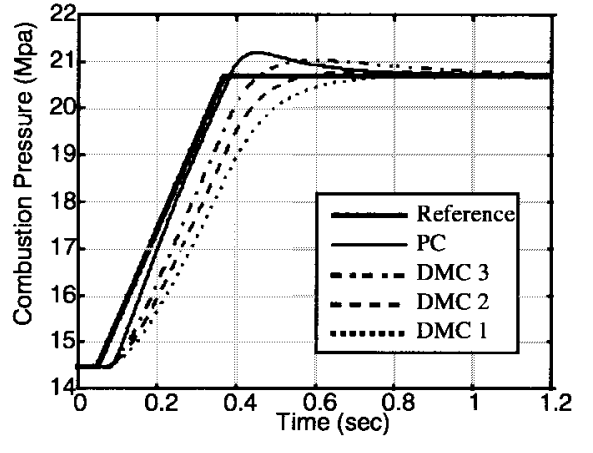

Fig. 3. Combustion chamber pressure performance.

\section{RESUlTS AND DisCUSSION}

This section presents the results of simulation experiments for rocket engine performance and structural durability under different controllers and different damage models. Fig. 2 shows the block diagram of the configuration used to simulate the closed-loop control system. The vector reference signal $y^{\text {ref }}$ is composed of the reference signals of the thrust chamber pressure and the $\mathrm{O}_{2} / \mathrm{H}_{2}$ mixture ratio. The reference signal for the chamber pressure is chosen such that the 6.21-MPa $(900$ $\left.\mathrm{lbf} / \mathrm{in}^{2}\right)$ swing in pressure range from $14.48 \mathrm{MPa}\left(2100 \mathrm{lbf} / \mathrm{in}^{2}\right)$ to $20.69 \mathrm{MPa}\left(3000 \mathrm{lbf} / \mathrm{in}^{2}\right)$ is realized in $1 / 3 \mathrm{~s}$ while that for the mixture ratio is held constant at 6.02. The feedforward control signal $u^{f f}$ is obtained at each sampling instant by interpolating the steady-state inputs corresponding to the initial and final states of the thrust chamber pressure. (Note that, unlike the previous work reported by [11], the feedforward signal in this paper is not optimized. This approach does not require computationally expensive nonlinear programming because the feedback controller is capable of compensating the tracking error.) The sampling interval is chosen as $0.002 \mathrm{~s}$ which is fast enough to capture the thermal-hydraulic dynamics of the rocket engine without any appreciable aliasing in the output signal.

Fig. 3 compares the performance of the four controllers, PC, $\mathrm{DMC} 1, \mathrm{DMC}$, and DMC3, for thrust chamber pressure. The $\mathrm{O}_{2} / \mathrm{H}_{2}$ mixture ratio remains within the acceptable limits of $6.02 \pm 0.08$ for both cases. The PC is supposed to generate best performance with no direct tradeoff with structural durability while the three damage-mitigating controllers, DMC1, DMC2, and DMC3, represent performance-damage tradeoff at differ- 

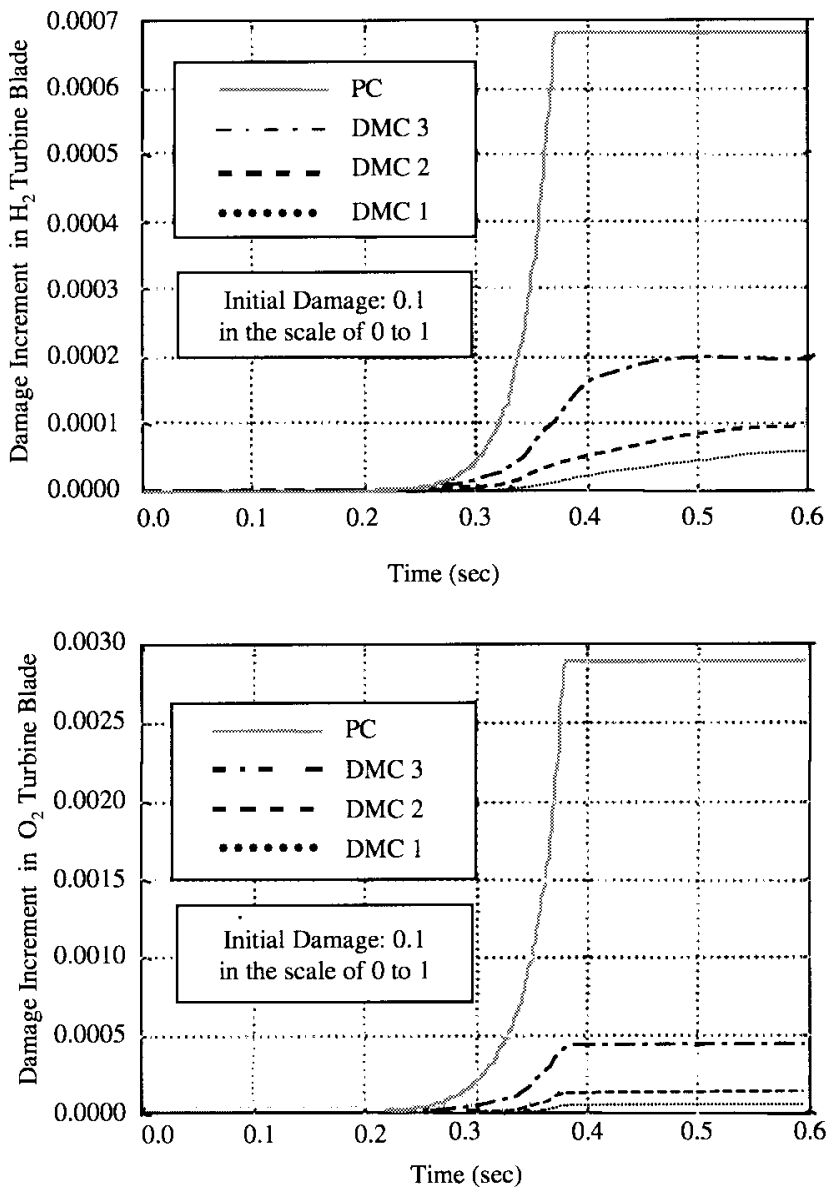

Strain-life Model

Fig. 4. Blade damage in hydrogen and oxygen turbines.

ent levels. Fig. 4 compares the fatigue damage increments in $\mathrm{H}_{2}$ and $\mathrm{O}_{2}$ turbine blades for each of the four closedloop systems by the strain-life-based damage model used in the earlier publication [5] and the state-space-based damage model currently used. The strain-life model predicts damage as a dimensionless abstract quantity (in the range of zero to one) while the state-space-based model predicts the physical crack length. Hence the results from the two models are not comparable in an absolute sense. However, one can convert the physical crack length into a dimensionless quantity relative to a specified critical crack length at which the service life of the component is expended. The plots in the left hand side of Fig. 4 show progressively larger reductions in blade damage increment predicted by the strain-life model as the rocket engine is controlled by PC, DMC1, DMC2, and DMC3. In contrast, the plots in the right-hand side of Fig. 4 do not show such trends in blade damage increment predicted by the state-space model under identical controllers.

The performance controller has the largest damage accumulation based on the strain-life model that does not adequately account for the effects of variable-amplitude stress. The three damage-mitigating controllers result in increasing amounts of damage accumulation with increasing chamber pressure performance. The corresponding results are not as expected according to the state-space model that does account for
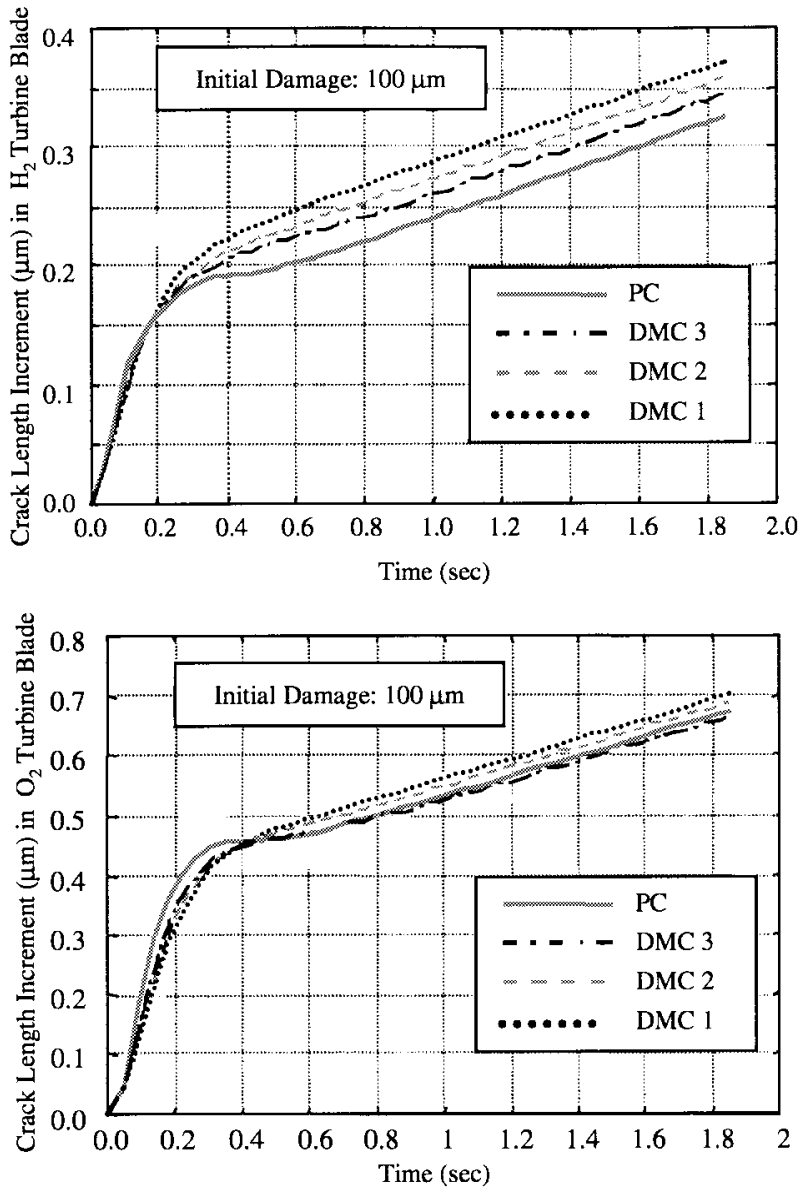

State-space Model variable-amplitude stress. In fact, the results could often be contrary to the expectations. The DMC controllers do not seem to be effective if structural durability of the critical components (i.e., $\mathrm{H}_{2}$ and $\mathrm{O}_{2}$ turbine blades) is measured in terms of fatigue damage based on the state-space model since the effects of stress-amplitude variations have a major bearing on the crack growth rate. The reason for the apparent paradox, described above, could be attributed to the dynamics of crack opening stress that directly influences the fatigue crack growth rate. The strain-life model does not adequately account for the dynamics of fatigue crack growth under variable-amplitude stress. In the earlier publication [4], the DMC controllers were designed based on the general notion that higher the peak stress, higher is the damage based on the strain-life model. This notion is true under constant-amplitude stress excitation but may not always hold under variable-amplitude stress.

Let us now carefully examine the fatigue crack growth, in $\mathrm{H}_{2}$ and $\mathrm{O}_{2}$ turbine blades, obtained from the state-space model for the closed-loop systems under PC and DMC's. After passing the peak load, the crack growth rate under PC is slowed down considerably as compared to those under DMC's as seen in Fig. 4. This crack growth retardation in PC lasts for about $0.2 \mathrm{~s}$ and crack growth rate slowly starts increasing again. Fig. 5(a) and (b) shows the variations in applied minimum and maximum stresses and their effects on 


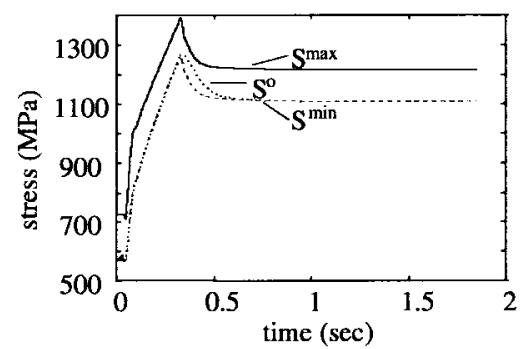

(a)

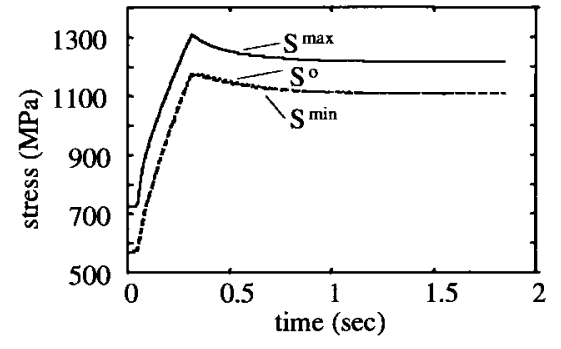

(b)

Fig. 5. Variations of crack opening stress in oxygen turbine blades.

$S^{\circ}$ for the oxygen turbine blade as a function of time for PC and DMC3, respectively. As both controllers display a sharp rise in applied stress for the first $0.32 \mathrm{~s}$, the peak stress is reached followed by a relaxation in stress. Then onwards, a constant amplitude stress is applied until the end of the maneuver. This constant-amplitude stress at the final stage is the same for all the controllers. The crack length rises sharply with the initial abrupt increase in applied stress but, after passing the peak stress, there is a significant retardation in crack growth for about $0.2 \mathrm{~s}$. This retardation effect is more prominent for the PC due to the stress overload [13]. The rationale for this phenomenon is attributed to the dynamics of the state variable $S^{o}$. After reaching the peak stress, when the stress continues to decrease, $S^{\circ}$ does not decrease instantaneously. The rate of decrease of $S^{\circ}$ is determined by the decay parameter $\eta$ in (4). According to (2), sustained higher values of $S^{o}$ result in a lower value of $\Delta K^{\text {eff }}$ which, in turn, results in lower crack growth during this period. Later on, as $S^{\circ}$ decays, the crack growth rate starts rising again. Since the peak stress reached under PC is higher than that under DMC3, the state variable $S^{\circ}$ reaches a higher value in PC and hence the crack growth retardation effect due to overload is more prominent under PC. The critical observation for DMC design is that the prolonged crack retardation effects of stress overload, normally ignored by the strain-life-based model, are well captured by the state-space model.

\section{SUMMARY AND CONCLUSIONS}

Since the goal of DMC is to achieve a tradeoff between structural durability and dynamic performance, accurate modeling of damage is one of the most important steps in the controller design procedure. So far DMC designers have used simplistic models for damage prediction. In the formulation of such models, it is often tacitly assumed that the fatigue crack damage resulting from constant-amplitude stress-excitation can be simply extrapolated to variable-amplitude stress excitation, thus completely ignoring the dynamical effects of the crack opening stress in fatigue crack growth. This brief paper demonstrates that the decisions of the DMC system, obtained by employing simplistic fatigue crack damage models, may not be reliable. Simulation results are presented to compare the predicted structural durability and closed-loop performance of a reusable rocket engine under the same controllers with two different damage models, with and without consideration of the effects of variable-amplitude cyclic stress.
The information on all state variables of the damage model must be incorporated into the control synthesis procedure to mitigate structural damage regardless of the actual performance trajectory. Simplistic damage models can only generate partial information of the damage variables that may not always be adequate for DMC design. As demonstrated by application of the state-space damage model to rocket engine turbine blades, expressing damage as a function of crack length alone is not sufficient under variable-amplitude stress. A damage measure needs to be defined in terms of both states of the damage model, viz., crack length and crack opening stress. Such a measure should account for the effects of stress overload on fatigue crack growth. Therefore, damage-mitigating controllers must be designed based on the dynamics of this damage measure under variable-amplitude stress excitation. Because of the highly nonlinear structure of the damage model, it might not be possible to design a linear damage-mitigating controller that will be effective under all kinds of variableamplitude stress excitations.

Finally, we conclude that a reliable damage model is a prerequisite for achieving tradeoffs between structural durability and dynamic performance. Specifically, several damagemitigating controllers may cause comparable damage in structural components. Under these circumstances, it may not be meaningful to choose a specific controller unless the damage model is appropriately experimentally verified.

\section{ACKNOWLEDGMENT}

The authors acknowledge technical contributions of their former colleague Dr. M. Holmes to this research. The authors are also grateful to Mr. C. F. Lorenzo of NASA Lewis Research Center for many stimulating technical discussions.

\section{REFERENCES}

[1] T. L. Anderson, Fracture Mechanics. Boca Raton, FL: CRC, 1995.

[2] B. A. Bamieh and J. B. Pearson, "A general framework for linear periodic systems with applications to sampled-data control," IEEE Trans. Automat. Contr., vol. 37, pp. 418-435, Apr. 1992.

[3] D. S. Dugdale, "Yielding of steel sheets containing slits," J. Mechanics, Phys., Solids, vol. 8, pp. 100-104, 1960.

[4] M. S. Holmes, S. Tangirala, and A. Ray, "Life-extending control of reusable rocket engines," J. Guidance, Contr., Dynamics, vol. 20, no. 3, pp. 621-623, 1997.

[5] M. S. Holmes and A. Ray, "Fuzzy damage mitigating control of mechanical structures," ASME J. Dynamic Syst., Measurement, Control, vol. 120, no. 2, pp. 249-256, June 1998. 
[6] C. F. Lorenzo and W. C. Merril, "Life extending control: A concept paper," in Proc. Amer. Contr. Conf., Boston, MA, June, 1991, pp. $1080-1095$.

[7] J. C. Newman, Jr., "A crack opening stress equation for fatigue crack growth," Int. J. Fracture, vol. 24, pp. R131-R135, 1984.

[8] —, "FASTRAN-II-A fatigue crack growth structural analysis program," Langley Research Center, NASA Tech. Memo 104159, 1992.

[9] R. P. Patankar, A. Ray, and A. Lakhtakia, "A state-space model of fatigue crack dynamics," Int. J. Fracture, vol. 90, no. 3, pp. 235-249, 1998.

[10] T. R. Porter, "Method of analysis and prediction for variable amplitude fatigue crack growth," Eng. Fracture Mechanics, vol. 4, pp. 717-736, 1972.
[11] A. Ray, M.-K. Wu, M. Carpino, and C. F. Lorenzo, "Damage-mitigating control of mechanical systems: Parts I and II," ASME J. Dynamic Syst., Measurement, Contr., vol. 116, no. 3, pp. 437-455, 1994.

[12] A. Ray and X. Dai, Damage-Mitigating Control of a Reusable Rocket Engine for High Performance and Extended Life, NASA Contractor Rep. 4640 prepared for NASA Lewis Research Center, 1995.

[13] J. Schijve, "Observations on the prediction of fatigue crack growth propagation under variable-amplitude loading," Fatigue Crack Growth under Spectrum Loads, ASTM STP 595, 1976, pp. 3-23.

[14] S. Suresh, Fatigue of Materials. Cambridge, U.K.: Cambridge Univ. Press, 1991.

[15] S. Tangirala, M. Holmes, A. Ray, and M. Carpino, "Life-extending control of mechanical structures: A feedforward-feedback approach," Automatica, vol. 34, no. 1, pp. 3-14, Jan. 1998. 